\title{
ASYMPTOTIC BEHAVIOUR OF SECOND ORDER NEUTRAL DIFFERENTIAL EQUATIONS WITH "MAXIMA"
}

\author{
D. BAINOV, V. PETROV AND V. PROYTCHEVA
}

\begin{abstract}
In the paper the asymptotic behaviour of nonoscillatory solutions of neutral equations with "maxima" is considered. Examples are given showing the difference between the equations with "maxima" and the corresponding neutral equations without "maxima".
\end{abstract}

\section{Introduction}

Consider the equation

$$
[x(t)+p(t) x(t-\tau)]^{\prime \prime}-q(t) \max _{[t-\sigma, t]} x(s)=0
$$

Though differential equations with "maxima" are often met in the applications, for instance in the theory of automatic control ([3], [4]), the qualitative theory of these equations is relatively little developed. The existence of periodic solutions of the equations with "maxima" is considered in [5] and [6]. The asymptotic stability of the solutions is investigated in [7]. The only paper in which the oscillatory properties of equations with "maxima" are considered is [1].

The main goal of the present paper is the investigation of the asymptotic behaviour of the nonoscillatory solutions of (1). An example is given showing the difference between equations with "maxima" and the corresponding equations without "maxima".

\section{Auxiliary Assertions}

We shall say that conditions $(\mathrm{H})$ are met if the following conditions hold. H1. $\tau, \sigma \in \mathbb{R}_{+}$

H2. $p(t) \in C\left(\left[t_{0}, \infty\right), \mathbb{R}\right)$

Received March, 9, 1994. 
H3. $q(t) \in C\left(\left[t_{0}, \infty\right), \mathbb{R}_{+}\right)$

H4. $\int_{i_{0}}^{\infty} q(t) d t=\infty$

By a solution of (1) we mean a continuous function $x$ on the interval $\left[t_{0}, \infty\right)$ such that $x(t)+p(t) x(t-\tau)$ is continuously differentiable and $x$ satisfies (1). As is customary, a solution of (1) is said to be oscillatory if it has arbitrarily large zeros, otherwise it is said to be nonoscillatory. In the sequel, for convenience, we will assume that inequalities concerning values of functions are satisfied eventually, that is for all large $t$. Define the function $z(t)$ as follows

$$
z(t)=x(t)+p(t) x(t-\tau)
$$

Then (1) implies that

$$
\begin{gathered}
z^{\prime \prime}(t)=q(t) \max _{[t-\sigma, t]} x(s) \\
z^{\prime}(t)=z^{\prime}\left(t_{0}\right)-\int_{t_{0}}^{t} q(s) \max _{[s-\sigma, s]} x(v) d s
\end{gathered}
$$

Lamma 1. Let conditions $(H)$ hold and

$$
p \leq p(t) \leq 0
$$

Then, if $x(t)$ is a positive solution of (1), then either

$$
z(t)>0, z^{\prime}(t)>0, z^{\prime \prime}(t) \geq 0 \text { and } \quad \lim _{t \rightarrow \infty} z(t)=\lim _{t \rightarrow \infty} z^{\prime}(t)=\infty
$$

or

$$
z(t)>0, z^{\prime}(t)<0, z^{\prime \prime}(t) \geq 0 \text { and } \quad \lim _{t \rightarrow \infty} z(t)=\lim _{t \rightarrow \infty} z^{\prime}(t)=0
$$

Proof. From (3) it follows that $z^{\prime \prime}(t) \geq 0$ and $z^{\prime}(t)$ is a nondecreasing function. On the other hand, H4 implies that $z^{\prime}(t) \not \equiv 0$ eventually. Thus either $z^{\prime}(t)>0$ or $z^{\prime}(t)<0$. Let $z^{\prime}(t)>0$. Since $z^{\prime}(t)$ is a nondecreasing function, $\lim _{t \rightarrow \infty} z(t)=\infty$. From (4) we obtain that $\lim _{t \rightarrow \infty} z^{\prime}(t)=\infty$. Hence if $z^{\prime}(t)>0$, then (6) is valid. Let $z^{\prime}(t)<0$. From the fact that $z^{\prime}(t)$ is a nondecreasing function it follows that there exists $\lim _{t \rightarrow \infty} z^{\prime}(t)=c \leq 0$. Suppose that $c<0$. Then $z^{\prime}(t)<c$ and $\lim _{t \rightarrow \infty} z(t)=-\infty$. From (2) it follows that the inequality

$$
z(t)>p(t) x(t-\tau)>p x(t-\tau)
$$

is valid and therefore $\lim _{t \rightarrow \infty} x(t)=\infty$. From (4) we obtain that $\lim _{t \rightarrow \infty} z^{\prime}(t)=-\infty$. The contradiction obtained shows that $\lim _{t \rightarrow \infty} z^{\prime}(t)=0$ and since $z^{\prime}(t)$ is a nondecreasing function, $z^{\prime}(t)<0$ and $z(t)$ is a decreasing function. Suppose that there exists the finite limit $\lim _{t \rightarrow \infty} z(t)=L$. Let $L>0$. The inequality $x(t)>z(t)$ implies that $x(t)>L$. 
From $H_{4}$ and (4) it follows that the relation $\lim _{t \rightarrow \infty} z^{\prime}(t)=\infty$ is valid and we get to a contradiction. Thus $L \leq 0$. Let $L<0$. The estimate

$$
\frac{L}{2}>z(t)=x(t)+p(t) x(t-\tau)>p(t) x(t-\tau)>p x(t-\tau)
$$

is valid. From the inequality $x(t-\tau)>\frac{L}{2 p}>0$ as above we obtain that $\lim _{t \rightarrow \infty} z^{\prime}(t)=$ $\infty$. Thus $L=0$ and since $z(t)$ is a decreasing function, then $z(t)>0$. Suppose that $\lim _{t \rightarrow \infty} z(t)=-\infty$. As above the inequality $x(t-\tau)>\frac{z(t)}{p}$ holds and $\lim _{t \rightarrow \infty} x(t)=\infty$. From (4) it follows that $\lim z^{\prime}(t)=\infty$ and we get to a contradiction. Thus if $z^{\prime}(t)<0$, then (7) is realized.

Lemma 2. Let conditions $(H)$ and (5) hold. Then, if $x(t)$ is a negative solution of (1), then either

$$
z(t)<0, z^{\prime}(t)<0, z^{\prime \prime}(t) \leq 0 \text { and } \lim _{t \rightarrow \infty} z(t)=\lim _{t \rightarrow \infty} z^{\prime}(t)=-\infty
$$

or

$$
z(t)<0, z^{\prime}(t)>0, z^{\prime \prime}(t) \leq 0 \quad \text { and } \quad \lim _{t \rightarrow \infty} z(t)=\lim _{t \rightarrow \infty} z^{\prime}(t)=0
$$

The proof of Lemma 2 is analogous to that of Lemma 1.

Lemma 3. The function $x(t)$ is a negative solution of equation (1) if and only if $-x(t)$ is a positive solution of the equation

$$
[y(t)+p(t) y(t-\tau)]^{\prime \prime}-q(t) \min _{[t-\sigma, t]} y(s)=0
$$

The assertion of Lemma 3 is verified immediately.

\section{Main results}

Theorem 1. Let conditions $(H)$ hold and

$$
p \leq p(t) \leq-1
$$

Then for each nonoscillatory solution $x(t)$ of (1) $\lim _{i \rightarrow \infty}|x(t)|=\infty$.

Proof. Let $x(t)<0$. Lemma 2 implies that (8) or (9) is valid. Suppose that (8) holds. Then from the inequality $x(t)<z(t)$ it follows that $\lim _{t \rightarrow \infty} x(t)=-\infty$ and the assertion of Theorem 1 is proved. Suppose that (9) is valid and let $c=\lim \sup _{t \rightarrow \infty} x(t)$. If $c<0$, then $x(t)<\frac{c}{2}$ and from (4) we obtain that $\lim _{t \rightarrow \infty} z^{\prime}(t)=-\infty$ which contradicts the relation $\lim _{t \rightarrow \infty} z^{\prime}(t)=0$ proved in Lemma 2. Hence $c=0$, i.e. $\lim _{\sup } \sin _{t \rightarrow \infty} x(t)=0$. There exists a sequence $\left\{t_{n}\right\}_{1}^{\infty}$ such that $\lim _{n \rightarrow \infty} t_{n}=\infty, \lim _{n \rightarrow \infty} x\left(t_{n}\right)=0$ and

$$
\max _{\left[t_{1}, t_{n}\right]} x(s)=x\left(t_{n}\right)
$$


On the other hand, since $z(t)<0$, then

$$
x(t)<-p(t) x(t-\tau) \leq x(t-\tau)
$$

But the inequality $x\left(t_{n}\right)<x\left(t_{n}-\tau\right)$ contradicts (11). Thus under the conditions of Theorem 1 of the two relations (8) and (9) only (8) is valid and $\lim _{t \rightarrow \infty} x(t)=-\infty$. The case when $x(t)>0$ is considered analogously.

Corollary 1. Let conditions $(H)$ and (10) hold. Then each bounded solution of (1) is oscillatory.

Theorem 2. Let conditions $(H)$ hold and

$$
-1<p \leq p(t) \leq 0
$$

Then, if $x(t)$ is a nonoscillatory solution of (1), then either $\lim _{t \rightarrow \infty} x(t)=0$ or $\lim _{t \rightarrow \infty}|x(t)|=\infty$

Proof. Let $x(t)>0$ and suppose that $x(t)$ is a bounded function. Obviously in this case of the two relations (6) and (7) only (7) is realized and thus $\lim _{t \rightarrow \infty} z(t)=$ 0 . Suppose that $c=\lim \sup _{t \rightarrow \infty} x(t)>0$. There exists a sequence $\left\{t_{n}\right\}^{\infty}$, such that $\lim _{n \rightarrow \infty} t_{n}=\infty$ and $\lim _{n \rightarrow \infty} x\left(t_{n}\right)=c$. Let $d=\limsup _{n \rightarrow \infty} x\left(t_{n}\right)$ (it is clear that $d \leq c)$. Choose a subsequence $\left\{n_{k}\right\} \subseteq\{n\}$ such that $\lim _{k \rightarrow \infty} x\left(t_{n_{k}}-\tau\right)=d$. From (2) and (12) the inequality

$$
z(t) \geq x(t)+p x(t-\tau)
$$

follows. We pass to the limit in the inequality

$$
z\left(t_{n_{k}}\right) \geq x\left(t_{n_{k}}\right)+p x\left(t_{n_{k}}-\tau\right)
$$

as $k \rightarrow \infty$ and obtain

$$
0 \geq c+p d \geq c+p c=(1+p) c>0
$$

The contradiction obtained shows that $\lim \sup _{t \rightarrow \infty} x(t)=0$ and $\lim _{t \rightarrow \infty} x(t)=0$. Let us assume that $x(t)$ is an unbounded solution of (1). We shall show that in this case relation (6) is valid. Suppose that this is not true. Since $x(t)$ is an unbounded function, there exists a sequence $\left\{\alpha_{n}\right\}$ such that $\lim _{n \rightarrow \infty} \alpha_{n}=\infty, \lim _{n \rightarrow \infty} x\left(\alpha_{n}\right)=\infty$ and $\max _{\left[\alpha_{1}, \alpha_{n}\right]} x(s)=x\left(\alpha_{n}\right)$. The following estimate is valid

$$
\begin{aligned}
z\left(\alpha_{n}\right) & =x\left(\alpha_{n}\right)+p\left(\alpha_{n}\right) x\left(\alpha_{n}-\tau\right) \geq x\left(\alpha_{n}\right)+p\left(\alpha_{n}\right) x\left(\alpha_{n}\right) \\
& =x\left(\alpha_{n}\right)\left(1+p\left(\alpha_{n}\right)\right) \geq x\left(\alpha_{n}\right)(1+p)
\end{aligned}
$$

Inequalities (12) imply that $\lim _{n \rightarrow \infty} z\left(\alpha_{n}\right)=\infty$ which contradicts the relation $\lim _{t \rightarrow \infty} z(t)=0$. Hence (6) is valid and $\lim _{t \rightarrow \infty} z(t)=\infty$. From the inequality 
$x(t)>z(t)$ it follows that $\lim _{t \rightarrow \infty} x(t)=\infty$. The case when $x(t)$ is a negative solution of (1) is considered analogously.

Theorem 3. Let conditions $(H)$ hold and

$$
0 \leq p(t) \leq p<1
$$

Then for each nonoscillatory solution of (1) either $\lim _{t \rightarrow \infty} x(t)=0$ or $\lim _{t \rightarrow \infty}|x(t)|=\infty$.

Proof. Let $x(t)>0$. From (3) it follows that $z^{\prime \prime}(t) \geq 0$ and $z^{\prime}(t)$ is a nondecreasing functions. H4 implies that either $z^{\prime}(t)>0$ or $z^{\prime}(t)<0$. Let $z^{\prime}(t)>0$. Obviously $\lim _{t \rightarrow \infty} z(t)=\infty$ and $z(t)$ is an increasing function. From (2) there follow the equalities

$$
\begin{aligned}
& z(t+\tau)=x(t+\tau)+p(t+\tau) x(t) \\
& p(t+\tau) z(t)=p(t+\tau) x(t)+p(t) p(t+\tau) x(t-\tau)
\end{aligned}
$$

From the above two equalities we obtain the equality

$$
z(t+\tau)-p(t+\tau) z(t)=x(t+\tau)-p(t) p(t+\tau) x(t-\tau)
$$

On the other hand,

$$
\begin{aligned}
& z(t+\tau)-p(t+\tau) z(t) \\
= & p(t+\tau)(z(t+\tau)-z(t))+(1-p(t+\tau)) z(t+\tau) \\
= & p(t+\tau) \tau z^{\prime}(\xi(t))+(1-p(t+\tau)) z(t+\tau)>(1-p) z(t+\tau)
\end{aligned}
$$

Hence the inequality

$$
x(t+\tau)>(1-p) z(t+\tau)
$$

is valid and since $\lim _{t \rightarrow \infty} z(t)=\infty$, then $\lim _{t \rightarrow \infty} x(t)=\infty$. Let $z^{\prime}(t)<0$. In this case $z(t)$ is a decreasing positive function. If $\lim _{t \rightarrow \infty} z^{\prime}(t)=c<0$ then $\lim _{t \rightarrow \infty} z(t)=-\infty$. Therefore $\lim _{t \rightarrow \infty} z^{\prime}(t)=0$. Suppose that $d=\lim _{t \rightarrow \infty} z(t)>0$ and consider the equality

$$
p(t+\tau) \tau z^{\prime}(\xi(t))+(1-p(t+\tau)) z(t+\tau)=x(t+\tau)-p(t) p(t+\tau) x(t-\tau)
$$

Since $\lim _{t \rightarrow \infty} z^{\prime}(t)=0$ and $\lim _{t \rightarrow \infty} \xi(t)=\infty$, then from the above equality it follows that for $t$ large enough the inequality $-\varepsilon+(1-p) d<x(t+\tau)$ is valid, where $\varepsilon$ is an arbitrarily small positive number. From the last inequality and (4) it follows that $\lim _{t \rightarrow \infty} z^{\prime}(t)=\infty$. The contradiction shows that $\lim _{t \rightarrow \infty} z(t)=0$. Then (2) implies the relation $\lim _{t \rightarrow \infty} x(t)=0$.

Theorem 4. Let conditions $(H)$ hold and $p(t) \equiv 1$.

Then if $x(t)$ is an unbounded nonoscillatory solution of $(1), \lim _{t \rightarrow \infty}|x(t)|=\infty$ 
Proof. Let $x(t)$ be a positive unbounded solution of (1). Then $z(t)$ is also an unbounded function. As above, either $z^{\prime}(t)>0$ or $z^{\prime}(t)<0$. If we assume that $z^{\prime}(t)<0$, then $z(t)$ is a decreasing positive function which contradicts the fact that it is unbounded. Thus $z^{\prime}(t)>0$ and $z(t)$ is an increasing function. Since $z^{\prime}(t)$ is increasing, there exists a positive constant $d$ such that $z^{\prime}(t) \geq d>0$. Define the function $w(t)$ as follows: $w(t)=z(t)-z(t-\tau)$. Then

$$
w(t)=x(t)-x(t-2 \tau)
$$

We sum up the equalities

$$
w(t+2 k \tau)=x(t+2 k \tau)-x(t+2(k-1) \tau), \quad k=1,2, \ldots, n
$$

and obtain

$$
\sum_{k=1}^{n} w(t+2 k \tau)=x(t+2 n \tau)-x(t)
$$

Let $\liminf \operatorname{in}_{t \rightarrow \infty} x(t)=c(0<c<\infty)$. Then the inequality $x(t)>\frac{c}{2}$ holds eventually. From the definition of $w(t)$ it follows that for each $k \in \mathbb{N}$ there exists $\alpha_{k}$ such that $\alpha_{k} \in[t+(2 k-1) \tau, t+2 k \tau]$ and $w(t+2 k \tau)=\tau z^{\prime}\left(\alpha_{k}\right)$. Therefore for $t \geq \bar{t}(\bar{t}$ is large enough) the inequality $w(t+2 k \tau) \geq d \tau$ holds. By virtue of the last inequality and (15) we obtain that for each $t \geq \bar{t}$ and each $n \in \mathbb{N}$ we have $x(t+2 n \tau) \geq n d \tau$. Choose a sequence $\left\{t_{k}\right\}_{k=1}^{\infty}$ such that $\lim _{k \rightarrow \infty} t_{k}=\infty$ and $\lim _{k \rightarrow \infty} x\left(t_{k}\right)=c$. Hence for sufficiently large $k$ the inequality $x\left(t_{k}\right)<2 c$ holds. Let $n \in \mathbb{N}$ be such that $n d \tau>2 c$. For that fixed $n$ choose the positive integer $m$ such that $t_{m}-2 n \tau>\bar{t}$. Set $\overline{\bar{t}}=t_{m}-2 n \tau$. Then $x(\overline{\bar{t}}+2 n \tau)=x\left(t_{m}\right)<2 c$. On the other hand, from the choice of $n$ and $\overline{\bar{t}}$ it follows that $x(\overline{\bar{t}}+2 n \tau)>n d \tau>2 c$. The contradiction obtained shows that either $c=0$ or $c=\infty$. Suppose that $c=0$, i.e. $\liminf _{t \rightarrow \infty} x(t)=0$. There exists a sequence $\left\{\beta_{n}\right\}_{1}^{\infty}$ such that $\lim _{n \rightarrow \infty} \beta_{n}=\infty, \lim _{n \rightarrow \infty} x\left(\beta_{n}\right)=0$ and $\min _{\left[\beta_{1}, \beta_{n}\right]} x(s)=x\left(\beta_{n}\right)$. Using the definition of $w(t)$ and the fact that $z(t)$ is an increasing function, we obtain that $w(t)>0$. Then (14) implies that $x(t)>x(t-2 \tau)$. This inequality, however, contradicts the relation $\min _{\left[\beta_{1}, \beta_{n}\right]} x(s)=x\left(\beta_{n}\right)$. Hence $\liminf _{t \rightarrow \infty} x(t)=\infty$ and $\lim x(t)=\infty$. The case when $x(t)$ is a negative solution of $(1)$ is considered analogously.

Theorem 5. Let conditions $\mathrm{H} 1-\mathrm{H} 3$ hold and $p(t) \equiv 1$. Let the function $q(t)$ also satisfy the condition:

$$
\text { H5. } \int_{t_{0}}^{\infty} \tilde{q}(t) d t=\infty, \quad \text { where } \tilde{q}(t)=\min \{q(t), q(t+\tau)\}
$$

Then for each bounded positive solution $x(t)$ of $(1) \lim _{t \rightarrow \infty} x(t)=0$

Proof. Since $x(t)>0$, then $z^{\prime \prime}(t) \geq 0$ and $z^{\prime}(t)$ is a nondecreasing function. H5 implies that $q(t) \not \neq 0$ eventually. Thus either $z^{\prime}(t)>0$ or $z^{\prime}(t)<0$. If $z^{\prime}(t)>0$, then 
$\lim _{t \rightarrow \infty} z(t)=\infty$, which contradicts the boundedness of $x(t)$. Hence $z^{\prime}(t)<0$ and $z(t)$ is a positive decreasing function. Let $c=\lim _{t \rightarrow \infty} z(t)$ and suppose that $c>0$. From (3) it follows that

$$
z^{\prime \prime}(t)+q(t-\tau) \max _{[t-\sigma-\tau, t-\tau]} x(s)=q(t) \max _{[t-\sigma, t]} x(s)+q(t-\tau) \max _{[t-\sigma-\tau, t-\tau]} x(s)
$$

Then, making use of the definition of $\tilde{q}(t)$ and of (2), we obtain that

$$
\begin{aligned}
z^{\prime \prime}(t)+q(t-\tau) \max _{[t-\sigma-\tau, t-\tau]} x(s) & \geq \tilde{q}(t-\tau)\left[\max _{[t-\sigma, t]} x(s)+\max _{[t-\sigma-\tau, t-\tau]} x(s)\right] \\
& =\tilde{q}(t-\tau)\left[\max _{[t-\sigma, t]} x(s)+\max _{[t-\sigma, t]} x(s-\tau)\right] \\
& \geq \tilde{q}(t-\tau) \max _{[t-\sigma, t]}(x(s)+x(s-\tau)) \\
& =\tilde{q}(t-\tau) \max _{[t-\sigma, t]} z(s)=\tilde{q}(t-\tau) z(t-\sigma)
\end{aligned}
$$

Since $z(t)$ is a decreasing function and $\lim _{t \rightarrow \infty} z(t)=c$, then $z(t)>c$ and the above inequality takes on the form

$$
z^{\prime \prime}(t)+q(t-\tau) \max _{[t-\sigma-\tau, t-\tau]} x(s)>c \tilde{q}(t-\tau)
$$

We integrate the last inequality from $t_{1}$ to $t$ and obtain

$$
\begin{aligned}
& z^{\prime}(t)-z^{\prime}\left(t_{1}\right)+\int_{t_{1}}^{t} q(s-\tau) \max _{[s-\tau-\sigma, s-\tau]} x(v) d s>c \int_{t_{1}}^{t} \tilde{q}(s-\tau) d s \\
& z^{\prime}(t)-z^{\prime}\left(t_{1}\right)+\int_{t_{1}-\tau}^{t-\tau} q(s) \max _{[s-\sigma, s]} x(v) d s>c \int_{t_{1}-\tau}^{t-\tau} \tilde{q}(s) d s
\end{aligned}
$$

Since $z^{\prime}(t)$ is a negative nondecreasing function, then $z^{\prime}(t)$ is a bounded function. On the other hand, H5 implies that the right-hand side of (16) tends to infinity as $t \rightarrow \infty$. Thus from (16) we obtain that

$$
\int_{t_{1}}^{\infty} q(t) \max _{[t-\sigma, t]} x(s) d t=\infty
$$

Integrating (3) from $t_{1}$ to $t$, we obtain the equality

$$
z^{\prime}(t)-z^{\prime}\left(t_{1}\right)=\int_{t_{1}}^{t} q(s) \max _{[s-\sigma, s]} x(v) d s
$$

Then (17) implies the relation $\lim _{t \rightarrow \infty} z^{\prime}(t)=\infty$. The contradiction obtained shows that $c=0$, i.e. $\lim _{t \rightarrow \infty} z(t)=0$. But from the inequality $x(t)<z(t)$ it follows immediately that $\lim _{t \rightarrow \infty} x(t)=0$ and the proof is complete. 
Remark 1 . Theorems 4 and 5 characterize completely the asymptotic behaviour of the positive nonoscillatory solutions of (1). In contrast to neutral equations without "maxima" the assertion of Theorem 5 is not valid for bounded negative solutions of (1) even under the stronger condition $q(t) \geq q>0$. We shall illustrate this fact by the following example.

Example 1. Consider the equation.

$$
\left[y(t)+y\left(t-\frac{1}{2}\right)\right]^{\prime \prime}-q(t) \min _{[t-1, t]} y(s)=0
$$

where

$$
q(t)=\left(1+e^{\frac{1}{2}}\right) e^{-t}\left(\min _{[t-1, t]}\left\{\varphi(s)+e^{-s}\right\}\right)^{-1}
$$

and $\varphi(t)$ is a 1-periodic function defined by the equality

$$
\varphi(t)= \begin{cases}t, & t \in\left[0, \frac{1}{2}\right] \\ 1-t, & t \in\left[\frac{1}{2}, 1\right]\end{cases}
$$

A straightforward verification yields that the function $y(t)=\varphi(t)+e^{-t}$ is a positive solution of equation (18). Furthermore, obviously $\liminf _{t \rightarrow \infty} y(t)=0$ and $\limsup _{t \rightarrow \infty} y(t)=$ $\frac{1}{2}$. On the other hand, the inequality

$$
e^{-t} \leq \min _{[t-1, t]}\left\{\varphi(s)+e^{-s}\right\} \leq e^{1-t}
$$

implies that $\frac{1+e^{1 / 2}}{2} \leq q(t) \leq 1+e^{1 / 2}$. Thus condition $H 5$ is valid (in fact, even the stronger condition $q(t) \geq q>0$ holds). Lemma 3 implies that although the conditions of Theorem 5 are met, equation (1) could have a negative bounded solution which does not tend to zero.

Theorem 6. Let conditions $(H)$ hold and

$$
1<p_{1} \leq p(t) \leq p_{2}
$$

Then if $x(t)$ is a bounded nonoscillatory solution of $(1)$, then $\lim _{t \rightarrow \infty} x(t)=0$.

Proof. Let $x(t)>0$. As in Theorem 5 it is proved that if $x(t)$ is a bounded positive solution of $(1)$, then $z^{\prime \prime}(t) \geq 0, z^{\prime}(t)<0$ and $z(t)>0$. Suppose that $d=$ $\liminf _{t \rightarrow \infty} x(t)>0$. Then $x(t)>\frac{d}{2}$. From this inequality and from (4) it follows that $\lim _{i \rightarrow \infty} z^{\prime}(t)=\infty$. The contradiction obtained shows that $\liminf _{t \rightarrow \infty} x(t)=0$. There exists a sequence $\left\{t_{n}\right\}_{1}^{\infty}$ such that $\lim _{t \rightarrow \infty} t_{n}=\infty$ and $\lim _{n \rightarrow \infty} x\left(t_{n}-\tau\right)=0$. Suppose that $c=\lim _{t \rightarrow \infty} z(t)>0$. Passing to the limit in the equality

$$
z\left(t_{n}\right)=x\left(t_{n}\right)+p\left(t_{n}\right) x\left(t_{n}-\tau\right)
$$


we obtain that $\lim _{n \rightarrow \infty} x\left(t_{n}\right)=c$. On the other hand,

$$
z\left(t_{n}+\tau\right)=x\left(t_{n}+\tau\right)+p\left(t_{n}+\tau\right) x\left(t_{n}\right)>p_{1} x\left(t_{n}\right)
$$

We pass to the limit in the inequality $z\left(t_{n}+\tau\right)>p_{1} x\left(t_{n}\right)$ and obtain $c \geq p_{1} c>c$. Hence $\lim _{t \rightarrow \infty} z(t)=0$ and since $x(t)<z(t)$, then $\lim _{t \rightarrow \infty} x(t)=0$. The case when $x(t)<0$ is considered analogously.

Acknowledgement. The present investigation was supported by the Bulgarian Ministry of Education and Science under Grant MM-7.

\section{References}

[1] D. D. Bainov, A. Zahariev, "Oscillating and asymptotic properties of a class of functional differential equations with maxima," Czechoslovak Math. J., 34 (1984), 247-251.

[2] I. Györi and G. Ladas, "Oscillation Theory of Delay Differential Equations," Clarendon Press, Oxford, 1991.

[3] A. R. Magomedov, "On some problems of differential equations with "maxima"," Izv. Acad. Sci. Azerb. SSR, Ser. Phys-Techn. and Math. Sci., 108, No. 1 (1977), 104-108 (in Russian).

[4] E. P. Popov, Automatic Regulation and Control, Nauka, Moscow, 1966 (in Russian).

[5] Yu. A. Ryabov, A. R. Magomedov, Differential equations with maxima. Preprint No. 75, Bacou, 1983, Inst. of Physics Acad. Sci. Azerb. SSR (in Russian).

[6] Yu. A. Ryabov, A. R. Magomedov, "On the periodic solutions of linear differential equations with maxima," Matil. Physics Acad. Sci. Ukr. SSR, No. 23 (1978), 3-9 (in Russian).

[7] H. D. Voulov, D. D. Bainov, "On the asymptotic stability of differential equations with "maxima"," Rend. Circ. Mat. Palermo, Serie II, Tomo XI, (1991), 385-420.

D. Bainov, Higher Medical Institute, Sofia and V. Petrov and V. Proytcheva, Technical University, Plovdiv. 